\title{
Familial, Adult Onset Form of \\ Leukoencephalopathy with Brain Stem and Spinal Cord Involvement: Inconstant High Brain Lactate and Very Slow Disease Progression
}

\author{
Pierre Labauge $^{\mathrm{a}} \quad$ Etienne Roullet ${ }^{\mathrm{b}}$ Odile Boespflug-Tanguy ${ }^{c}$ François Nicolid, $\mathrm{e}$ \\ Yann Le Fur $^{d}$ Patrick J. Cozzone ${ }^{d}$ Denis Ducreux ${ }^{f}$ Diana Rodriguez $^{g}$ \\ aService de Neurologie, CHU Montpellier-Nîmes, Hôpital Caremeau, Nîmes, b Service de Neurologie and CITESEP, \\ Hôpital Tenon, AP-HP, Université Pierre et Marie Curie, Paris, ' Service de Génétique Médicale, \\ CHU de Clermont-Ferrand, INSERM UMR 384, Faculté de Médecine, Université Clermont 1, Clermont-Ferrand, \\ ${ }^{\mathrm{d} C e n t r e}$ de Résonance Magnétique Biologique et Médicale, UMR-CNRS 6612, Faculté de Médecine, Université \\ de la Méditerranée, and Hôpital de la Timone, ${ }^{e}$ Service d'Urgences Neuro-vasculaires, CHU Timone, Marseille, \\ ${ }^{f}$ AP-HP, Service de Neuroradiologie, et Inserm UMR 788, CHU de Bicêtre, Le Kremlin-Bicêtre, and ' ${ }^{\text {Service de }}$ \\ Neuropédiatrie, Hôpital Armand Trousseau, AP-HP, INSERM U546, Faculté de Médecine Pitié Salpétrière, \\ Paris, France
}

Dear Sir,

White matter (WM) disorders represent a diagnostic challenge for neurologists. During the last two decades, magnetic resonance (MR) patterns recognition has helped in the classification of this heterogeneous group of disorders to identify the genes involved. A phenotype, characterized by an ataxic paraparesis, related to a leukoencephalopathy with brain stem and spinal cord involvement on MR imaging (MRI) and high lactate content on MR spectroscopy (MRS) have been individualized as LBSL syndrome [1]. We report a familial, adult onset form in 2 siblings with an intrafamilial variability in brain lactate and a very slow disease progression over a 30-year follow-up.

\section{Description of the Family}

A 40-year-old woman (case 1) was referred because of progressive ataxic gait and mild spasticity since the age of 20. Examination showed spastic paraparesis with bilateral Babinski signs, proprioceptive ataxia, distal symmetrical decrease in position and vibration sense. The patient was able to walk $1,000 \mathrm{~m}$ without assistance. Cognitive functions were normal. MRI showed extensive WM lesions characterized by the abnormal signals of selective tracts in the supratentorial regions (fig. 1a), the brain stem, the cerebellum (fig. 1b, c) and the spinal cord (fig. 1d, e). Using chemical shift imaging MRS [2] (echo time $=135 \mathrm{~ms}$ ) lactate signal was never detected. Ratios of the resonance area of each detected metabolite including $\mathrm{N}$-acetyl aspartate (NAA), choline-containing compounds $(\mathrm{CHO})$, creatinephosphocreatine over a particular metabolite were calculated. In voxels centered on the paraventricular WM lesions, NAA/ $\mathrm{CHO}$ ratios were decreased in comparison with age-matched healthy controls (1.46 vs. $1.83 \pm 0.19)$ while $\mathrm{CHO} /$ creatinephosphocreatine ratios were increased ( 1.65 vs. $1.35 \pm 0.24$, respectively). MRS was normal in the cortex. Follow-up of this patient (age 49) showed mild worsening of the motor disability $(600 \mathrm{~m}$ ambulation without aid) and appearance of right trigeminal neuralgia. MRI findings were un- changed except for the disappearance of WM cerebellar hyperintensities (data not shown). Mild ataxic gait was noticed in her elderly brother (case 2) since the age of 15 , with a very slow increase of the symptoms. At the age of 50, neurological examination as well as MRI patterns (fig. 1f, g) were identical with the index case including mild motor disability (500 $\mathrm{m}$ ambulation) and right trigeminal neuralgia. Chemical shift imaging showed normal metabolic ratios, but a slight lactate signal in the centrum semiovale. Within the spinal cord lesions, the isotropic apparent diffusion coefficient was increased with slightly decreased fractional anisotropy values (fig. 1h). Metabolic screening (vitamins E and $\mathrm{B}_{12}$, folic acid, lactate, pyruvate, very long chain fatty acids, phytanic acid, lysosomal enzymes, urine organic acids, oligosacchariduria, sulfatiduria) as well as sequencing of the coding regions of GFAP and EIF2B 1-5 genes were normal. Both nonconsanguineous parents (age 76 and 77) had normal clinical and brain-spinal MR.

\section{KARGER}

Fax +4161306 1234 E-Mail karger@karger.ch www.karger.com www.karger.com/ene
Prof. Pierre Labauge

Service de Neurologie, CHU Montpellier-Nîmes

Hôpital Caremeau, 2 Avenue du Pr Debré

FR-30029 Nîmes cedex 4 (France)

Tel. +334666832 61, Fax +334666840 16, E-Mail labauge@yahoo.fr 

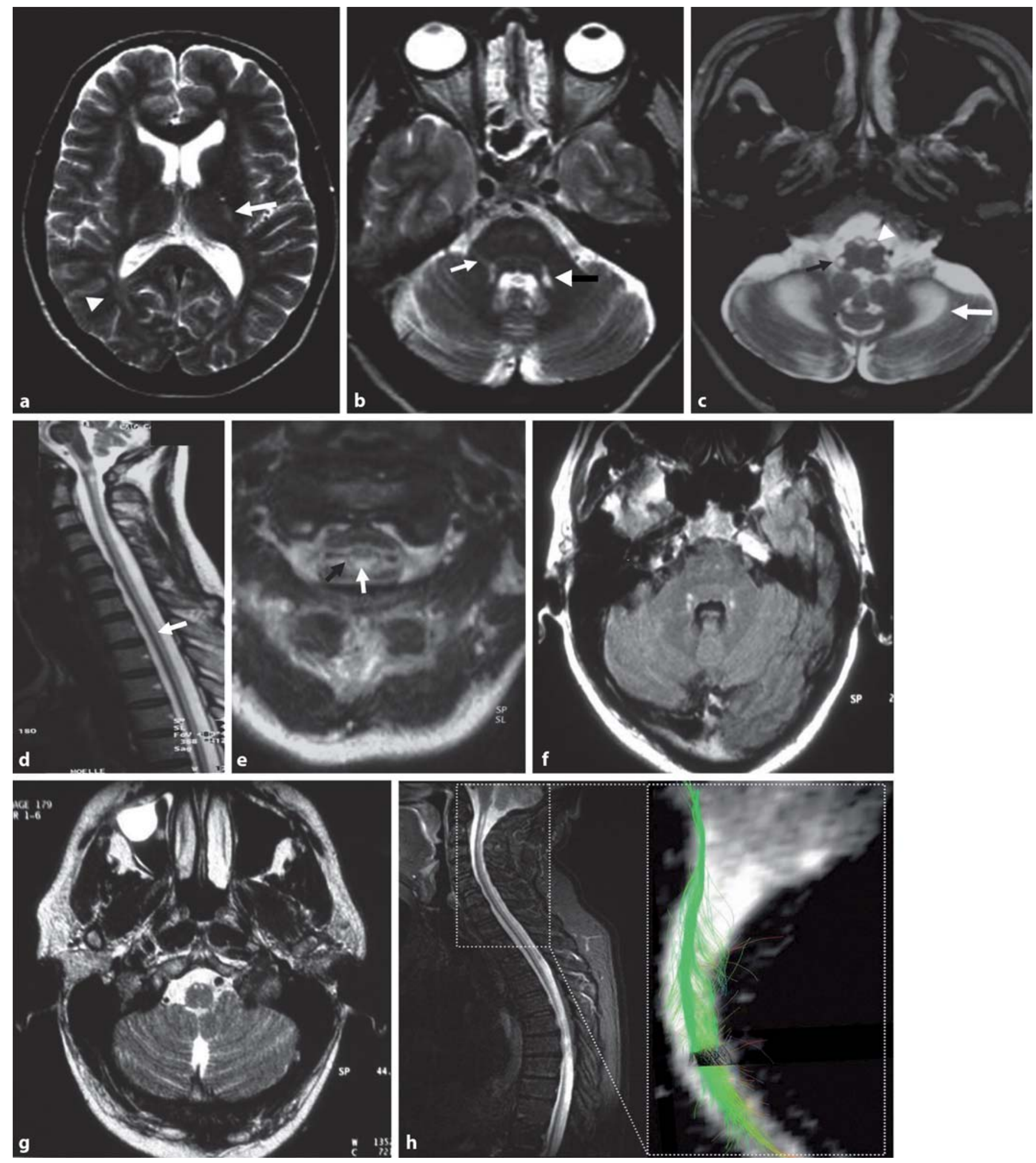


\section{Discussion}

After the initial LBSL description [1] in 8 children, 12 patients from 3 additional reports [3-5] confirmed this clinico-MR entity. As previously suggested $[1,5]$, the genetic origin of this syndrome is confirmed by the occurrence of 2 affected siblings in our family. Absence of clinical and MR signs in both parents argues for recessive inheritance. However, the rarity of reported affected siblings and consanguinity cannot rule out a dominant de novo mutation with germinal mosaicism as described for GFAP mutations.

Our report underlines the possible extension of disease onset to young adulthood. The variable brain lactate content observed even after a long evolution confirmed that high lactate cannot be considered as a major diagnostic criterium of LBSL [5]. Absence of increased lactate levels on MRS has been suggested to be secondary to the long evolution of the disease [5]. This explanation cannot be retained since our patients have the same evolution of the disease. High lactate has to be considered as a secondary event, since it has been reported in several other WM diseases (multiple sclerosis, Krabbe and Alexander diseases). The long-term follow-up of our patients (30 years) demonstrated for the first time a very slow disease progression at least in this adult onset forms with involvement of myelinated as well as nonmyelinated tracts and mild NAA decrease. Fiber tracking of these cervical spinal cord showed the particular severe alteration of the cordonal posterior fibers. However, trigeminal nerve involvement in both patients showed that the disease process also involved brainstem fibers of a peripheral origin. Disappearance of cerebellar WM abnormalities on serial MR of one of our patients associated with absence of atrophy and the slow progression of the disease is unusual in a disorder where a primary axonal degeneration has been suspected [1]. These features will not be elucidated until the genetic defect of the disease has been identified.

\section{Acknowledgements}

The authors thank Monique Petit, Florence Niel (Biochimie Médicale, CHU, Clermont-Ferrand, France), Fernande Gauthier-Barrichard, Laetitia Horzinski, Lydie Burglen (Unité de Génétique, Hôpital Armand Trousseau, Paris, France) for their technical help in GFAP and EIF2B 1-5 sequencing. This work has been supported (O.B.T. and D.R.) by the European Leukodystrophy Fundation (ELA 04).
Fig. 1. MR patterns in case 1. a-e Brain $\mathrm{T}_{2}$-weighted axial images. a Hyperintense signals involving periventricular area (arrowhead) and the posterior limbs of the internal capsule (white arrow). b Hyperintense signal of the superior cerebellar peduncles (white arrowhead), intraparenchymal and mesencephalic trajectories of the trigeminal nerves (white arrow). c Hyperintense signal of the inferior cerebellar peduncles (black arrow), pyramidal tracts (white arrowhead), and cerebellar WM (white arrow). d Spinal cord
$\mathrm{T}_{2}$-weighted sagittal image: hyperintense signal of the dorsal part of the cervical and thoracic spinal cord (white arrow). e Spinal cord $\mathrm{T}_{2}$-weighted axial incidence: hyperintense signal of the dorsal column (white arrow) and lateral corticospinal tracts (black arrow). f Brain FLAIR axial image. Hyperintense signal of the intraparenchymal trigeminal nerve and superior cerebellar peduncles. g Brain $\mathrm{T}_{2}$ weighted axial image. Hyperintense signals of the two pyramidal tracts. $\mathbf{h}$ Spinal cord STIR sagittal image (left) with

\section{References}

1 Van der Knaap MS, van der Voorn P, Barkhof F, et al: A new leukoencephalopathy with brainstem and spinal cord involvement and high lactate. Ann Neurol 2003;53:252-258.

2 Galanaud D, Le Fur Y, Nicoli F, et al: Regional metabolite levels of the normal posterior fossa studied by proton chemical shift imaging. MAGMA 2001;13:127-133.

3 Linnankivi T, Lundbom N, Autti T, et al: Five new cases of a recently described leukoencephalopathy with high brain lactate. Neurology 2004;63:688-692.

4 Serkov SV, Pronin IN, Bykova OV, et al: Five patients with a recently described novel leukoencephalopthy with brainstem and spinal cord involvement and elevated lactate. Neuropediatrics 2004;35:1-5.

5 Petzold GC, Bohner G, Klingebiel R, et al: Adult onset leucoencephalopathy with brain stem and spinal cord involvement and normal lactate. J Neurol Neurosurg Psychiatry 2006;77:889-891. zoomed fiber tracking reconstructions on the cervical spinal cord (right). Hyperintense signal of the pyramid tract and dorsal part of the cervical spinal cord on the STIR sequences, with altered pattern in number and shape of the cordonal posterior fibers on fiber tracking. Within the WM lesions, the isotropic apparent diffusion coefficient was increased (1.623 \pm $0.021 \times 10^{-9} \mathrm{~m}^{2} \cdot \mathrm{s}^{-1}$ vs. $1.01 \pm 0.009 \times$ $10^{-9} \mathrm{~m}^{2} \cdot \mathrm{s}^{-1}$ in healthy control subjects), with slightly decreased FA values (0.58 \pm 0.08 vs. $0.74 \pm 0.04)$. 